\title{
Can phenology and plasticity prevent adaptive clines in tolerance limits across temperate mountains?
}

\author{
Luis Gutiérrez-Pesquera ${ }^{1}$, Miguel Tejedo ${ }^{2}$, Agustin Camacho ${ }^{3}$, Urtzi Enríquez-Urzelai ${ }^{4}$, \\ Marco Katzenberger ${ }^{5}$, Magdalena Choda ${ }^{6}$, Pol Pintanel ${ }^{1}$, and Alfredo Nicieza ${ }^{6}$ \\ ${ }^{1}$ Estación Biológica de Doñana \\ ${ }^{2}$ Affiliation not available \\ ${ }^{3}$ Universidade de São Paulo \\ ${ }^{4}$ Institute of Vertebrate Biology Czech Academy of Sciences \\ ${ }^{5}$ Universidade Federal de Pernambuco \\ ${ }^{6}$ University of Oviedo
}

August 6, 2021

\begin{abstract}
Critical thermal limits (CTmax and CTmin) are predicted to decrease with elevation, with greater change in CTmin, and the risk to suffer heat and cold stress increasing at the gradient ends. A central prediction is that populations will adapt to the prevailing climatic conditions. Yet, reliable support for such expectation is scant because of the complexity of integrating phenotypic and molecular divergence. We propose that phenotypic plasticity and breeding phenology may hinder local adaptation cancelling the appearance of adaptive patterns. We examined intraspecific variation of CTmax/CTmin in 11 populations of an amphibian across an elevational gradient, and assessed (1) the existence of local adaptation through a PST-FST comparison, (2) the acclimation scope in both thermal limits, and (3) the vulnerability to suffer acute heat (CTmax-tmax) and cold (tmin-CTmin) thermal stress, measured at both macro- and microclimatic scales. Our study revealed significant microgeographic variation in CTmax/CTmin, and unexpected elevation gradients in pond temperatures. However, variation in CTmax/CTmin could not be attributed to selection because critical thermal limits were not correlated to elevation or temperatures. Differences in breeding phenology among populations resulted in exposure to higher and more variable temperatures at mid and high elevations. Accordingly, mid- and high-elevation populations had higher CTmax and CTmin plasticities than lowland populations, but not more extreme CTmax/CTmin. Thus, we confirm our prediction that plasticity and phenological shifts may hinder local adaptation, promoting thermal niche conservatism and a higher vulnerability to climate change. This contradicts some of the existing predictions on adaptive thermal clines.
\end{abstract}

\section{Hosted file}

Guti\selectlanguage\{ngerman\}érrez-Pesquera et al 2021_V9_23 JULI0 2021 def.pdf available at https://authorea.com/users/429402/articles/533116-can-phenology-and-plasticity-preventadaptive-clines-in-tolerance-limits-across-temperate-mountains 